\title{
TRIM28 knockdown increases sensitivity to etoposide by upregulating E2F1 in non-small cell lung cancer
}

\author{
LEI LIU ${ }^{1}$, LIJUN XIAO ${ }^{1}$, XIUJUN LIANG ${ }^{2}$, LONG CHEN $^{2}$, LUYANG CHENG ${ }^{1}$, \\ LEI ZHANG ${ }^{3}$, XIAOGUANG WU ${ }^{2}$, QIAN XU ${ }^{2}$ and CHUNHU MA ${ }^{4}$ \\ Departments of ${ }^{1}$ Immunology and ${ }^{2}$ Central Laboratory, Basic Medical Institute, Chengde Medical College; \\ ${ }^{3}$ Department of Community Care, Nursing Institute, Chengde Medical College; \\ ${ }^{4}$ Clinical Skills Center, Chengde Medical College, Chengde, Hebei 067000, P.R. China
}

Received October 23, 2016; Accepted May 2, 2017

DOI: $10.3892 /$ or.2017.5638

\begin{abstract}
Tripartite motif containing 28 (TRIM28) is a universal corepressor for Kruppel-associated box zinc finger proteins. In our previous study, it was shown that expression of TRIM28 is upregulated in non-small cell lung cancer (NSCLC) cell lines and tissues. Here, we demonstrated that the stable silencing of TRIM28 expression by a specific siRNA lentivirus vector increased the sensitivity of NSCLC cells to chemotherapeutic agent etoposide. Combination of TRIM28 siRNA and etoposide significantly inhibited the growth and proliferation of lung adenocarcinoma PAa cells and exerted obvious antitumor effects in nude mice. Using FCM and terminal deoxynucleotidyl transferase (TdT)-mediated dUTP nick-end labeling (TUNEL) assay, we found that TRIM28 siRNA in combination with etoposide increased apoptosis in vitro and in vivo which was induced by E2F1 activity, since the expression of E2F1 and its target genes was significantly increased in the cotreatment group. Cell proliferation and apoptosis were almost completely abolished in the PAa cells cotreated with TRIM28 siRNA and etoposide following knockdown of E2F1. The results of our study demonstrated that the combination of TRIM28 siRNA and etoposide may be effective against NSCLC and has the potential of being a new therapeutic tool for future treatment.
\end{abstract}

\section{Introduction}

Lung cancer is the leading cause of cancer-related deaths worldwide, and non-small cell lung cancer (NSCLC) constitutes the predominant histologic type, representing $80 \%$ of all

Correspondence to: Professor Lei Liu, Department of Immunology, Basic Medical Institute, Chengde Medical College, Anyuan Road, Shuangqiao, Chengde, Hebei 067000, P.R. China

E-mail: homingreceptor@hotmail.com

Key words: NSCLC, TRIM28, etoposide, E2F1, small interfering RNA, apoptosis, proliferation, PAa, nude mice lung neoplasias (1). The vast majority of patients are diagnosed at a locally advanced stage or with distant metastasis (2). For these unresectable patients, the standard treatment modality is two cycles of chemotherapy with etoposide and cisplatin followed by radiation. However, the prognosis for a large portion of patients remains poor. The median survival time for patients treated with chemoradiotherapy is approximately 15 months, and the 5-year survival rate is only $5-17 \%$ (3). Therefore, it is urgent to find novel and more effective therapeutic targets with which to improve the prognosis of advanced NSCLC patients.

Tripartite motif containing 28 (TRIM28) is an important member of the tripartite motif superfamily that regulates embryonic development (4), chromatin organization, erythroblast differentiation (5), and the DNA damage response (6-9), acting as an essential corepressor for KRAB zinc finger proteins. Resent studies have shown that it also plays a role in carcinogenesis and metastasis of cervical cancer (10), breast cancer (11), colorectal cancer (12), gastric cancer (13) and Kaposi's sarcoma (14). Our previous study also demonstrated that TRIM28 promoted the proliferation and metastatic progression of NSCLC and may be a new marker with which to predict metastasis and prognosis in early stage NSCLC patients (15). Wang et al (16) reported that TRIM28 stimulated formation of the E2F1-HDAC1 complex and inhibited E2F1 acetylation, acting as a partial backup to prevent E2F1-mediated apoptosis in the absence of retinoblastoma protein (pRb). Yet, the underlying mechanism of TRIM28 in NSCLC remains unclear.

Etoposide is one of the alternative first-line chemotherapeutic agents for NSCLC. Moreover, the sensitivity of tumor cells to etoposide is correlated with the induction of E2F1. Overexpression of E2F1 protein in tumor cell lines was found to lead to enhanced cytotoxicity following exposure to DNA damaging agent etoposide (17). Hence, in this study, whether the combination of TRIM28 siRNA and etoposide can augment apoptosis in vitro and in vivo was investigated, since TRIM28 contributes to the negative regulation of E2F1 which has been shown to be critical for etoposide-induced apoptosis. In addition, the possible underlying mechanisms involved in the tumorigenesis of NSCLC regulated by TRIM28 were examined. 


\section{Materials and methods}

Cell culture and RNA interference assay. Human NSCLC cell line PAa (BioVector NTCC Inc., Shanghai, China) was grown in monolayer in Dulbecco's modified Eagle's medium (DMEM) supplemented with $10 \% \mathrm{FCS}$ and was maintained at $37^{\circ} \mathrm{C}$ in an atmosphere of humidified air with $5 \% \mathrm{CO}_{2}$. Complementary oligonucleotides containing short hairpin RNA (shRNA) targeting TRIM28 was dimerized and cloned into the pLVTHM lentiviral vector (GeneChem, Shanghai, China). The targeting sequence for TRIM 28 shRNA construct was: 5'-CAC TGAGGACTACAACCTT-3, sense and 5'-GCGATCTGGTT ATGTGCAA-3, antisense. A replication-incompetent lentivirus was produced by co-transfection of the pLVTHM/ TRIM28-shRNA expression vector and ViraPower packaging mix containing an optimized mixture of two packaging plasmids: psPAX2 and pMD2.G into 293T cells. PAa cells were infected with lentiviral particles containing specific or negative control vectors, and the polyclonal cells with strong GFP expression were selected by flow cytometric sorting using anti-GFP monoclonal antibody (Abcam, Cambridge, UK) to culture and expand.

Ten micrograms of E2F1 siRNA expression vector and negative control vector (GeneChem) were transfected using $30 \mathrm{ml}$ of Lipofectamine 2000 (Invitrogen, Carlsbad, CA, USA) into PAa cells. The target sequences of the synthetic oligonucleotides for RNAi were as follows: siRNA-E2F1 sense, 5'-GACGUGUCAGGACCUUCGU-3' and antisense, 5'-ACG AAGGUCCUGACACGUC-3'. The transfected cells were cultured for $48 \mathrm{~h}$, and mRNA expression and protein level in the cells were confirmed by RT-PCR and western blot analysis, respectively.

Real-time quantitative PCR assay. After infection for 1, 2, 3 and 4 days, total RNA was extracted from blank control, PAa/ control-siRNA (si-control) and PAa/TRIM28-siRNA cells using TRIzol reagent (Invitrogen) according to the manufacturer's protocol. cDNA was synthesized from $2 \mu \mathrm{g}$ of total RNA using a Reverse transcription kit (Clontech Laboratories, Inc., Palo Alto, CA, USA). The PCR reaction included the following components: $100 \mathrm{nmol} / \mathrm{l}$ of each primer, diluted cDNA templates, and SYBR-Green PCR Master Mix (Biosystems, Warrington, UK), to a total volume of $20 \mu \mathrm{l}$. The real-time quantitative PCR assays were conducted using an ABI Prism 7000 Sequence Detection System instrument (Biosystems Life Technologies, Foster City, CA, USA) under the following conditions: $94^{\circ} \mathrm{C}$ for 4 min followed by 40 cycles at $94^{\circ} \mathrm{C}$ for $15 \mathrm{sec}, 58^{\circ} \mathrm{C}$ for $30 \mathrm{sec}$ and $72^{\circ} \mathrm{C}$ for $35 \mathrm{sec}$. All reactions were performed in triplicate and GAPDH mRNA was used as the internal control. The relative quantity of mRNA, normalized against GAPDH mRNA, was expressed in terms of cycle threshold $(\mathrm{Ct})$ using the following equations: $2^{\text {-(Ct TRIM28 - Ct GAPDH) }}$. The primer sequences for TRIM28 gene were: sense, 5'-ATGTGAGCGTGTACTGCTGG-3' and antisense, 5'-ACGTCTGCCTTGTCCTCAGT-3' (Shanghai Shenggong Co., Ltd., Shanghai, China).

Western blot analysis. Blank control, PAa/control-siRNA and PAa/TRIM28-siRNA cells were lysed in ice-cold lysis buffer: $50 \mathrm{mmol} / \mathrm{l}$ Tris- $\mathrm{HCl}$ (pH 8.0), $150 \mathrm{mmol} / \mathrm{l} \mathrm{NaCl}, 0.5 \% \mathrm{NP} 40$,
$0.5 \%$ sodium deoxycholate, $0.1 \%$ SDS, plus protease inhibitor (Calbiochem, Darmstadt, Germany). The proteins were separated by sodium dodecyl sulphate-polyacrylamide gel electrophoresis (SDS-PAGE) and transferred to polyvinylidene difluoride membranes (Millipore, Bedford, MA, USA). The membranes were blocked with $0.5 \%$ milk in phosphate-buffered saline (PBS) for $1 \mathrm{~h}$ at room temperature. After being washed in Tris-buffered saline Tween (TBST), the membranes were incubated for $1 \mathrm{~h}$ at room temperature with an appropriate dilution of rabbit polyclonal TRIM28 antibody (ProteinTech Inc., Chicago, IL, USA). After being washed in TBS-T, the blots were incubated with horseradish peroxidase-conjugated goat anti-rabbit IgG for $1 \mathrm{~h}$ and exposed to X-ray film at room temperature. The signal was detected by chemiluminescence using ECL detection kit (Amersham, Amersham, UK).

Cytotoxicity assay. To determine the $\mathrm{IC}_{50}$ value of etoposide, the PAa cells were treated with various concentrations of etoposide $(0.001,0.2,0.5,1,2$ and $4 \mu \mathrm{M})$. The cell group treated with only $1 \%$ DMSO (solvent of etoposide) was considered as the etoposide blank control. Twenty-four hours later, the cytotoxicity of the treatments was assessed using 3-(4, 5-dimethylthiazol-2-yl)-2, 5-diphenyltetrazolium bromide (MTT) cell proliferation kit (Roche Diagnostics $\mathrm{GmbH}$, Mannheim, Germany) according to the manufacturer's protocol. Absorbance at $570 \mathrm{~nm}$ was measured using an ELISA plate reader (Bio-Tek Instruments, Menlo Park, CA, USA). The survival rate (SR) was determined using the following equation: SR $(\%)=($ A treatment $/ \mathrm{A}$ control $) \times 100 \%$. The concentration that produced $50 \%$ cytotoxicity $\left(\mathrm{IC}_{50}\right)$ was determined by GraphPad Prism 6.02 software (GraphPad Software Inc., San Diego, CA, USA).

The effect of TRIM28 siRNA on the chemosensitivity of NSCLC cells was also determined using MTT assay. In 96-well plates, the four cell groups: PAa/control-siRNA, PAa/TRIM28-siRNA, PAa and PAa/TRIM28-siRNA, were divided into 500 cells/well in $100 \mu \mathrm{l}$. After $16 \mathrm{~h}$, the third and the fourth cell groups were treated with etoposide ( $\mathrm{IC}_{50}$ dose) in a 96-well plate and incubated at 1, 2,3 and 4 days. After using the MTT cell proliferation kit, the amount of formazan dye was determined by quantifying its absorbance (A) at $570 \mathrm{~nm}$.

Soft agar assay. PAa cells were divided into four groups. The first and second cell groups were transfected with TRIM28siRNA and control-siRNA, respectively. Then, the cells $\left(1 \times 10^{4}\right)$ were resuspended in DMEM containing 10\% fetal bovine serum (FBS) with $0.3 \%$ agarose and layered on top of $0.6 \%$ agar in medium supplemented with $20 \% \mathrm{FBS}$ on 60 -mm plates. The plates were incubated at $37^{\circ} \mathrm{C}$ in a humid atmosphere of $5 \% \mathrm{CO}_{2}$. The third group of PAa cells and the fourth cell group of cells transfected with TRIM28-siRNA were incubated in DMEM containing etoposide ( $\mathrm{IC}_{50}$ dose). After 3 weeks, cell colony numbers were counted under a microscope and cell colonies were photographed at an original magnification of $\mathrm{x} 100$. Only cell colonies containing more than 50 cells were counted. The experiment was performed for three independent times for each group.

Tumorigenicity in nude mice. This study was conducted in 40 female BALB/c nude mice (Vital River, Beijing, China) 
at 4 weeks of age, which were divided into four groups of 10 animals. The first and the second group of mice were subcutaneously injected with PAa/control-siRNA and PAa/TRIM28-siRNA cells, respectively. The third group of mice received a single i.v. injection of etoposide solution at $80 \mathrm{mg} / \mathrm{kg}$. The i.v. injections were administered via a tail vein at a rate of $\sim 0.05 \mathrm{ml} / \mathrm{sec}$, using a 27 -gauge needle and syringe. The fourth group of mice were subcutaneously injected with PAa/TRIM28-siRNA cells and then received an i.v. injection of etoposide solution. Mice were observed daily for viability and changes in general health and behavior. Body weights were determined once a week. Tumor onset was scored visually and by palpitation at the sight of injection by two trained laboratory staff at different times on the same day. Average tumor size was estimated by physical measurement of the excised tumor at the time of sacrifice. With the exception of mice with large tumor burdens, animals were sacrificed 4 weeks after injection. Protocols were conducted under the supervision of an authorised investigator following approval of the Institutional Ethics Committee of Chengde Medical College (Chengde, Hebei, China).

Flow cytometric analysis. Cells were washed twice with PBS, and incubated with $5 \mu \mathrm{l}$ Annexin V-Fluos and $5 \mu \mathrm{lPI}$ solution (Roche Diagnostics $\mathrm{GmbH}$ ) in $1 \mathrm{X}$ binding buffer (10 mM HEPES, pH 7.4, $140 \mathrm{mM} \mathrm{NaCl}, 2.5 \mathrm{mM} \mathrm{CaCl}_{2}$ ), at a final concentration of $1 \times 10^{6}$ cells $/ 100 \mu \mathrm{l}$ solution for $15 \mathrm{~min}$. After incubation, the cells were analyzed by flow cytometry. A minimum of 10,000 cells were analyzed using a FACSAria II flow cytometer (BD Biosciences, San Jose, CA, USA). Data were analyzed using FlowJo (Tree Star, Inc., Ashland, OR, USA) flow cytometry analysis software.

TUNEL assay. Apoptosis was quantitatively analyzed by detection of DNA fragmentation via a fluorescence assay based on terminal deoxynucleotidyl transferase (TdT)-mediated dUTP nick-end labeling (TUNEL) technique (Beyotime Institute of Biotechnology, Beijing, China). This method takes advantage of DNA fragmentation, characteristic of apoptosis. The DNA breaking points (nicks) expose the 3 'OH ends of DNA, which are labeled, thus allowing the identification of apoptotic cells. Briefly, terminal deoxynucleotidyl transferase was used to incorporate residues of digoxigenin nucleotide into the $3 \mathrm{OH}$ ends of DNA fragments. Immunohistochemical procedures for detecting apoptotic cells were performed according to the manufacturer's instructions. For each slide, 5 fields were randomly chosen under a microscope for each section. The apoptosis index (AI) was determined as: $\mathrm{AI}=$ number of positively stained apoptotic cells/total number of cells counted $\mathrm{x}$ 100. Assays were performed in a blinded manner.

Immunohistochemistry. Immunohistochemical analyses for TRIM28 and E2F1 were performed on formalin-fixed, paraffin-embedded surgical sections obtained from the 4 mouse groups by using the streptavidin-peroxidase method (ZhongShan Golden Bridge Biotech Co., Ltd., Beijing, China). Slides were deparaffinized and rehydrated with xylene and graded alcohol. Optimal antigen retrieval was carried out in citrate buffer ( $\mathrm{pH}$ 6.0) for $10 \mathrm{~min}$ with a steam oven to enhance the immunoreactivity. Primary rabbit polyclonal antibodies against TRIM28 and E2F1 (ProteinTech Inc., Chicago, IL, USA) was used at a dilution of 1:100.

Statistical analysis. Data are presented as mean \pm standard deviation (SD). Analysis of variance (ANOVA) followed by Bonferroni's test was used to determine significant differences between groups. Values of $\mathrm{P}<0.05$ were considered significant. All statistical analyses were performed using GraphPad Prism software.

\section{Results}

TRIM28 siRNA in combination with etoposide inhibits the growth of NSCLC cell line PAa. Our previous study indicated that the expression of TRIM28 was significantly higher in 8 NSCLC cell lines in comparison with bronchial epithelia BEAS2B cells (15). TRIM28-specific siRNA lentivirus vector and a negative control vector (si-control) were designed and constructed to silence TRIM28 in lung adenocarcinoma PAa cells, which express higher TRIM28 than other NSCLC cell lines. Relative TRIM28 gene expression was calculated in relation to the blank control group, which was considered as $100 \%$. Quantitative RT-PCR analysis and western blot assay were used to confirm that TRIM28 siRNA resulted in a marked time-dependent reduction in both TRIM28 mRNA and protein levels $(\mathrm{P}<0.05$, relative to the blank control). At $1,2,3$ and 4 days post-transfection, the relative expression of TRIM28 mRNA was 53.67 $\pm 6.51(\mathrm{P}=0.000), 36.00 \pm 6.08$ $(\mathrm{P}=0.000), 24.67 \pm 6.43(\mathrm{P}=0.000)$ and $14.00 \pm 3.61 \%(\mathrm{P}=0.000)$, respectively (Fig. 1A), while the relative TRIM28 protein expression levels were 64.67 $\pm 4.16(\mathrm{P}=0.000), 45.00 \pm 3.00$ $(\mathrm{P}=0.000), 36.67 \pm 1.53(\mathrm{P}=0.000)$ and $19.33 \% \pm 2.08(\mathrm{P}=0.000)$, respectively (Fig. 1B and C). Notably, treatment with siRNA control had no effect on TRIM28 mRNA $(\mathrm{P}=0.829)$ and protein expression compared to the blank control $(\mathrm{P}=0.291)$. To determine the $\mathrm{IC}_{50}$ dose of etoposide, PAa cells treated with variable concentrations of etoposide were analyzed using MTT assay. As shown in Fig. 1D, monotreatment with etoposide induced cytotoxicity in a dose-dependent manner and the $\mathrm{IC}_{50}$ value of etoposide was $2.30 \mu \mathrm{M}$.

To investigate whether the combination of TRIM28 siRNA and etoposide affects the proliferation of NSCLC cells, we compared the proliferation of PAa cells treated with control siRNA, TRIM28 siRNA, etoposide, and combination of TRIM28 siRNA and etoposide using an MTT assay. As shown in Fig. 1E, PAa cells cotreated with TRIM28 siRNA and etoposide exhibited a significant growth inhibition when compared with the cells treated with the other methods. Compared with PAa/control-siRNA cells, the NSCLC cells treated with TRIM28 siRNA, etoposide and the combination of TRIM28 siRNA and etoposide exhibited $33.78 \pm 1.67(\mathrm{P}=0.000), 37.84 \pm 2.84(\mathrm{P}=0.000)$ and $67.57 \pm 1.69 \%$ $(\mathrm{P}=0.000)$ decreased cell proliferation by day 4 , respectively. Furthermore, the combination group achieved a statistically significant difference compared to the TRIM28 siRNA group $(\mathrm{P}=0.000)$ or etoposide group $(\mathrm{P}=0.000)$.

The effect of growth inhibition was further examined by a soft agar, a test for colony formation ability. As shown in Fig. 1F, the ability of PAa cells to grow in soft agar was significantly inhibited by TRIM28 siRNA, etoposide, and 

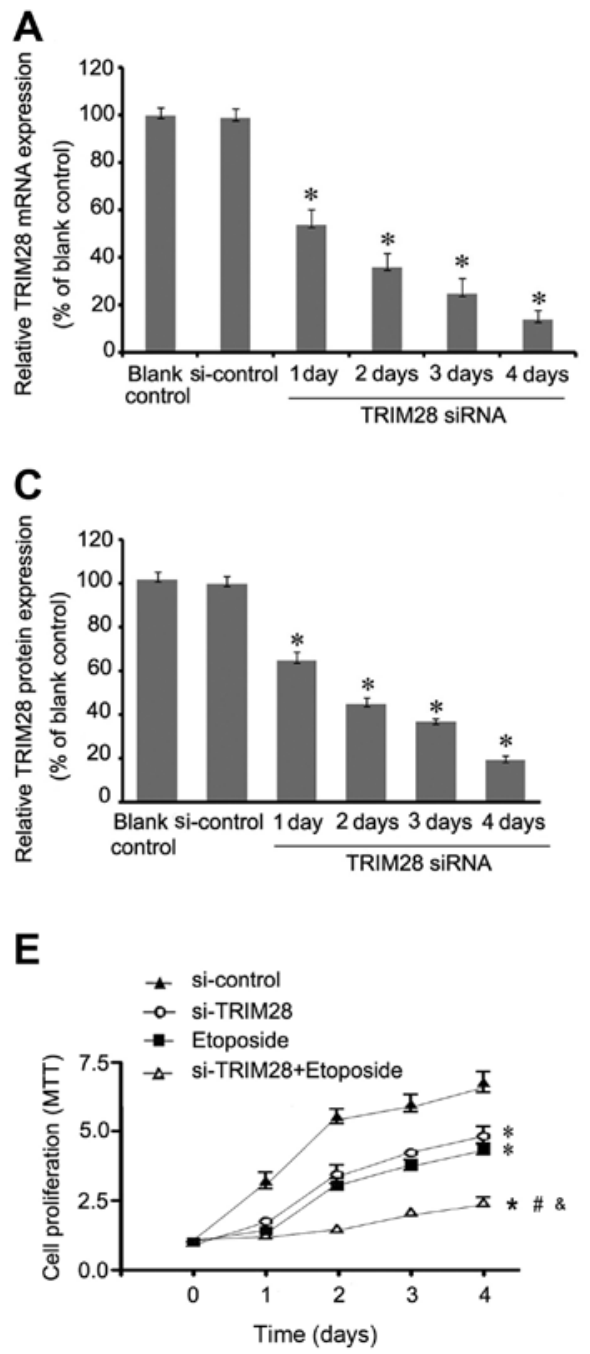

B

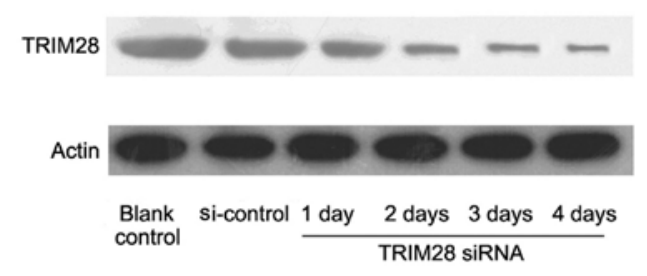

$\mathbf{D}$

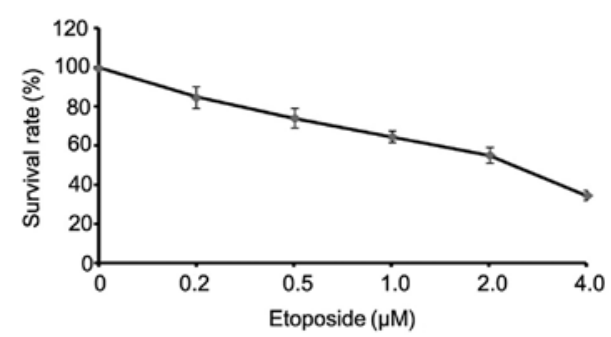

$\mathbf{F}$

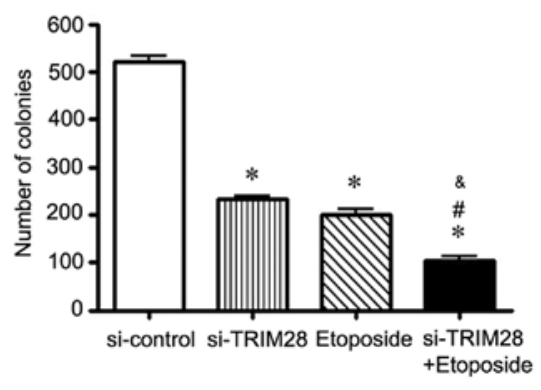

Figure 1. TRIM28 siRNA in combination with etoposide inhibits the growth of non-small cell lung cancer (NSCLC) PAa cells. (A and B) TRIM28 expression was silenced by specific siRNA lentivirus vector and negative control vector in PAa cells at 1,2,3 and 4 days. TRIM28 mRNA and protein levels were examined by real-time PCR (A) and western blot analysis (B and C). (B) Representative western blot analysis of TRIM 28 and $\beta$-actin proteins from cells transfected with siRNAs. (C) The expression level of each band was measured by densitometry and normalized to the corresponding $\beta$-actin. The data represent mean $\pm \mathrm{SD}(\mathrm{n}=3) ;{ }^{*} \mathrm{P}=0.000$ vs. blank control. (D) The cytotoxic effect of etoposide was determined by MTT assay and the $50 \%$ inhibitory concentration $\left(\mathrm{IC}_{50}\right)$ of etoposide was $2.30 \mu \mathrm{M}$. (E) Effects of control-siRNA, TRIM28 siRNA, etoposide or the combination of TRIM28 siRNA and etoposide on NSCLC PAa cell proliferation were measured for 1, 2, 3, and 4 days by MTT assay. (F) Quantification of the numbers of colonies in the colony formation assay of PAa cells treated with control-siRNA, TRIM28 siRNA, etoposide or the combination of TRIM28 siRNA and etoposide. Values represent mean \pm SD from three independent experiments. ${ }^{*} \mathrm{P}=0.000$ in comparison to si-control group. ${ }^{\text { }} \mathrm{P}=0.000$ in comparison to TRIM28 siRNA group. ${ }^{\text {\& }} \mathrm{P}=0.000$ in comparison to etoposide group.

cotreatment compared with control siRNA. In soft agar, PAa cells treated with TRIM28 siRNA and etoposide formed $242 \pm 7(\mathrm{P}=0.000)$ and $221 \pm 19$ colonies $(\mathrm{P}=0.000)$, respectively. The cells treated with the combination of TRIM28 siRNA and etoposide formed the least number of colonies $(144 \pm 16)$ $(\mathrm{P}=0.000)$, while those treated with control siRNA formed $520 \pm 15$ colonies.

Antitumor effect of TRIM28 siRNA in combination with etoposide on tumor growth in nude mice. Mice received an injection of PAa/TRIM28-siRNA cells alone and in combination with $80 \mathrm{mg} / \mathrm{kg}$ etoposide for 4 weeks (5 times/ week) and tumor growth was observed during the treatment period. Growth inhibition was observed with TRIM28 siRNA alone or etoposide alone, and this inhibition was statistically significant when compared with that of the control $(\mathrm{P}=0.000)$.
It appeared that etoposide treatment was more effective than injection of PAa/TRIM28-siRNA. Moreover, the combination of TRIM28 siRNA and etoposide treatment was more effective than either method alone ( $\mathrm{P}=0.000$; Fig. 2A-C). No loss of body weight was observed in mice treated with PAa/ TRIM28-siRNA and/or etoposide during the experimental period (Fig. 2D).

TRIM28 siRNA in combination with etoposide increases apoptosis in vitro and in vivo. After the NSCLC cell line PAa was cotreated with TRIM28 siRNA and etoposide, we observed some abnormal changes. PAa cells underwent a shrinkage in cell size and cells became round and detached from the plates, which suggested that cell apoptosis had occurred. To analyze the degree of apoptosis, flow cytometry was performed. As shown in Fig. 3A and B, apoptosis was observed in $0.97 \pm 0.27 \%$ 
A

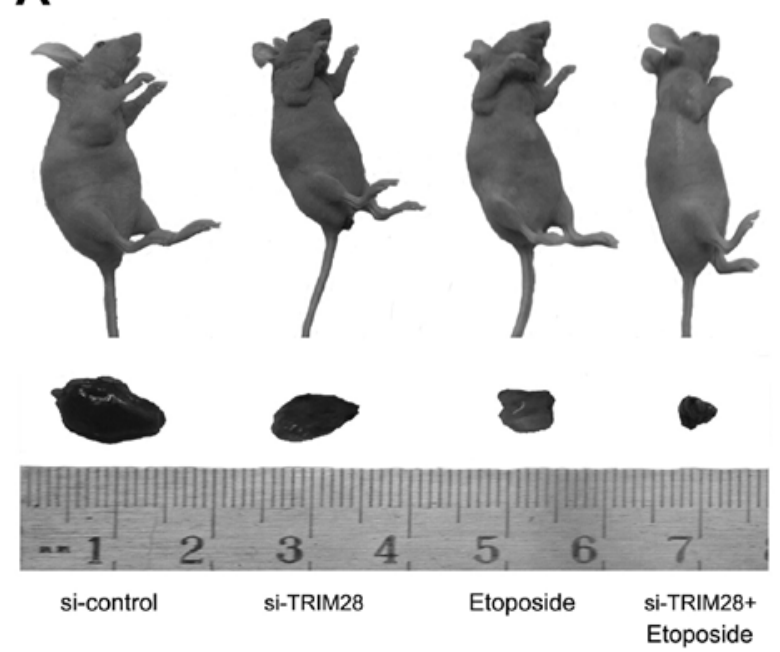

B

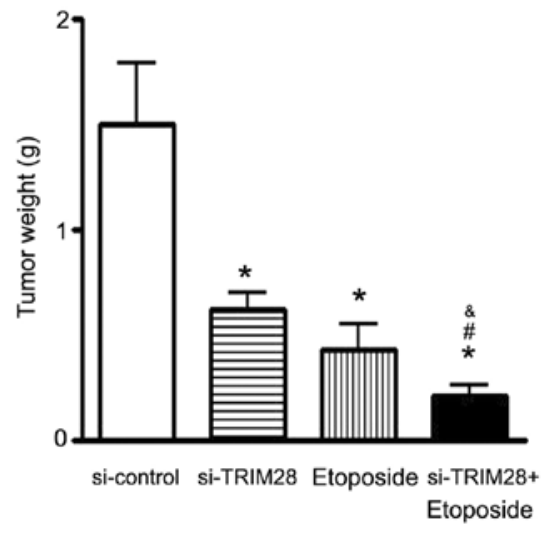

C

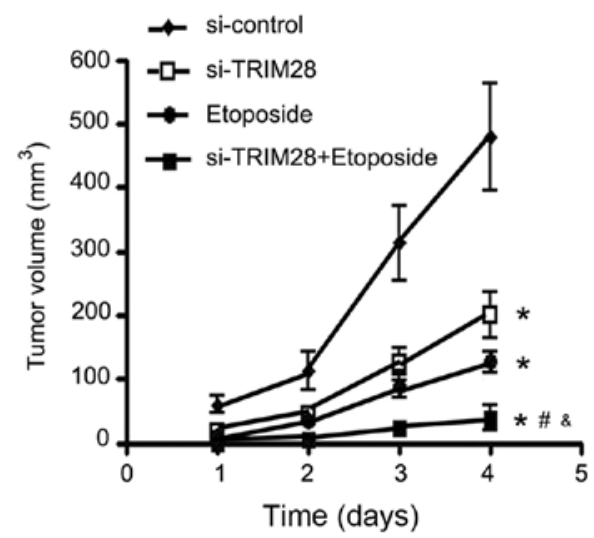

D

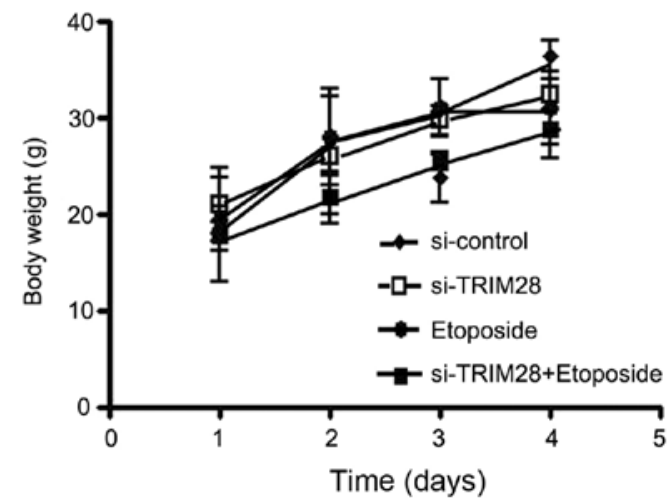

Figure 2. Tumor growth is inhibited by the combination of TRIM28 siRNA and etoposide in nude mice. (A) Top: images of representative mice with tumors from the four groups. Bottom: images of excised tumors from each group. (B) Tumors from four mouse groups were dissected after 4 weeks of treatment, and the tumor weight was recorded. (C) Tumor growth curve showed a significant growth inhibition in the TRIM28 siRNA plus etoposide group. (D) During the experimental period no loss of body weight was observed in the mice. ${ }^{*} \mathrm{P}=0.000$ in comparison to the si-control group. ${ }^{\#} \mathrm{P}=0.000$ in comparison to the si-TRIM28 group. ${ }^{\&} \mathrm{P}=0.000$ in comparison to the etoposide group.

of the PAa cells transfected with control siRNA, $3.03 \pm 0.25 \%$ $(\mathrm{P}=0.001)$ of the PAa cells transfected with TRIM28 siRNA and $3.26 \pm 0.31 \%(\mathrm{P}=0.001)$ of the cells treated with etoposide, while more apoptotic cells were observed in the cells treated with the combination of TRIM28 siRNA and etoposide $(8.21 \pm 0.22 \%, \mathrm{P}=0.000)$.

To further confirm whether the combination of TRIM28 siRNA and etoposide promoted tumor cell apoptosis, tumors were removed from mice after treatment and the number of apoptotic cells was quantified by TUNEL assay. The degree of apoptosis in the tumors treated with either TRIM28 siRNA or etoposide alone was significantly higher than that observed in the control group. The largest number of apoptotic cells was observed in the tumors cotreated with TRIM28 siRNA and etoposide. The combination treatment significantly induced apoptosis compared to each treatment alone as well as that noted in the control (Fig. 3C). The extent of apoptosis (the percentage of total TUNEL-positive cells) in the groups were as follows: control siRNA group, $8.20 \pm 2.11$; TRIM28 siRNA group, $24.65 \pm 4.05(\mathrm{P}=0.004)$; etoposide group, $27.8 \pm 4.87$
( $\mathrm{P}=0.004)$; TRIM28 siRNA plus etoposide group, 43.94 \pm 3.18 $(\mathrm{P}=0.000)$ (Fig. 3D).

TRIM28 siRNA in combination with etoposide increases the expression of E2F1. To test the hypothesis that E2F1 may play a role in both TRIM28 siRNA and etoposide-induced cell death, the expression of E2F1 protein and several E2F1 target genes was examined following treatment of PAa with control siRNA, TRIM28 siRNA, etoposide or the combination of TRIM28 siRNA and etoposide. As shown in Fig. 4A, reduction in the TRIM28 level or treatment with etoposide resulted in increased expression of E2F1, cyclin D3, P27, dihydrofolate reductase (DHFR) and $\mathrm{Bcl}-2$. This combination treatment significantly induced the expression of E2F1 and its target proteins, compared to each treatment alone as well as to the control.

Tumors from mice were removed and processed for further histologic and immunohistochemical analyses. We found that E2F1 expression was upregulated in the TRIM28 siRNA and etoposide treatment groups compared with that in the 
A

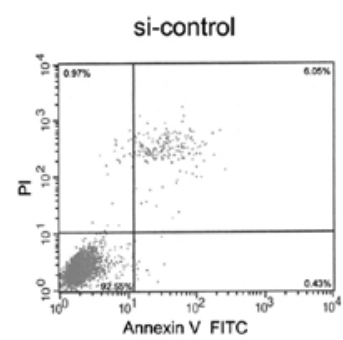

Etoposide
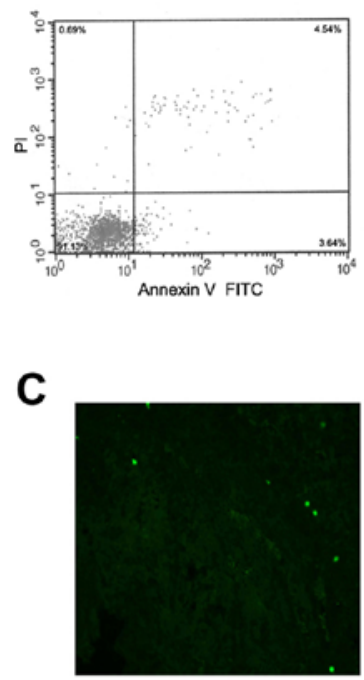

si-control

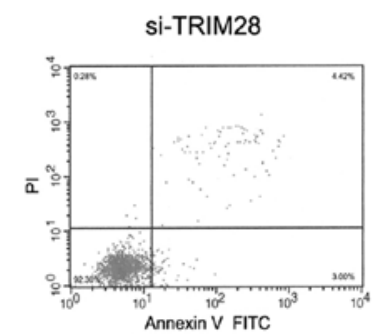

si-TRIM28+Etoposide
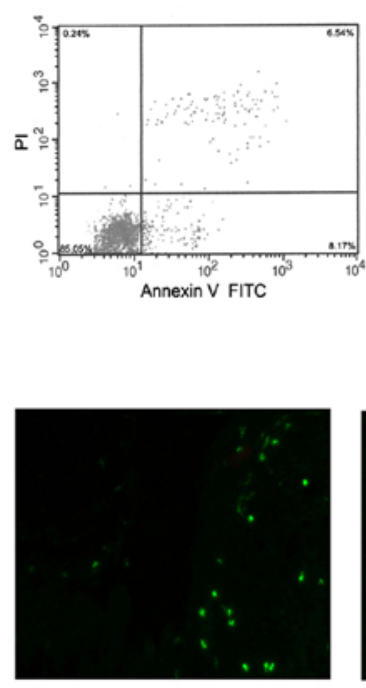

si-TRIM28
B
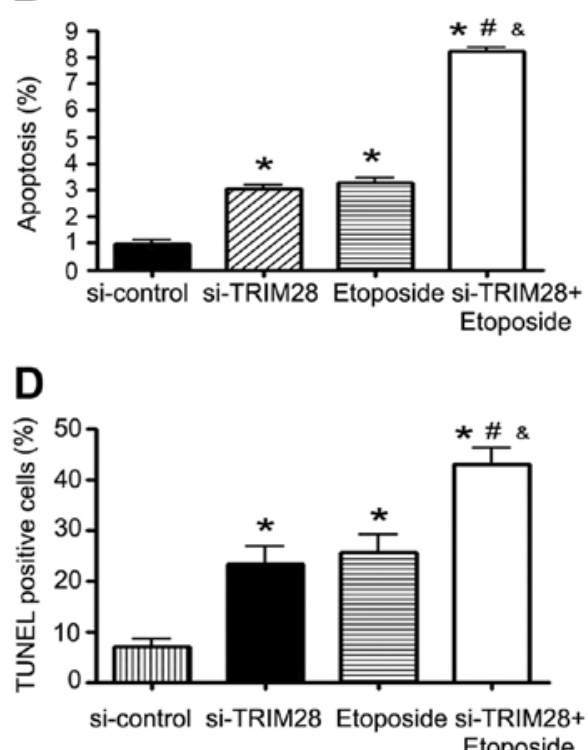

Etoposide

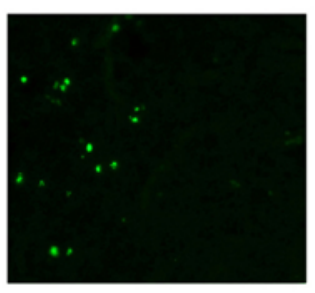

Etoposide

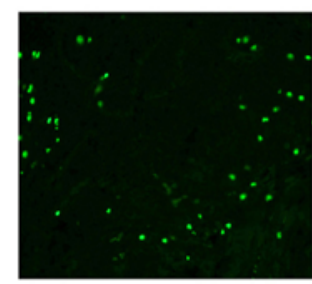

si-TRIM28 + Etoposide

Figure 3. TRIM28 siRNA in combination with etoposide promotes apoptosis. (A and B) Flow cytometric analysis indicating the apoptotic PAa cells treated with negative control siRNA, TRIM28 siRNA, etoposide or the combination of TRIM28 siRNA and etoposide. (C and D) Terminal deoxynucleotidyl transferase (TdT)-mediated dUTP nick-end labeling (TUNEL) assay (original magnification x200). Analysis of the degree of apoptosis present in tumors from the mice treated with control siRNA, TRIM28 siRNA, etoposide or the combination of TRIM28 siRNA and etoposide. The largest number of apoptotic cells was noted in the tumors treated with the combination of TRIM 28 siRNA and etoposide. ${ }^{*} \mathrm{P}<0.05$ in comparison to the si-control group. ${ }^{\#} \mathrm{P}<0.05$ in comparison to the si-TRIM28 group. ${ }^{\&} \mathrm{P}<0.05$ in comparison to the etoposide group.

control siRNA group, while the tumor cells in the cotreatment group had significantly higher levels of E2F1 expression as compared with those in the other treatment groups or the control group (Fig. 4B). As shown in Fig. 4C, the percentage of E2F1-positive cells from 10 random fields in the combination group was increased by $65.24 \pm 3.82 \%(\mathrm{P}=0.003)$, compared to that in the control siRNA group.

E2F1 mediates the sensitivity to etoposide which is enhanced by TRIM28 siRNA. In order to determine whether the increased sensitivity of PAa cells to etoposide after treatment with TRIM28 siRNA depends on E2F1, we examined the cell proliferation and apoptosis in PAa cells transfected with TRIM28 siRNA alone or together with E2F1 siRNA and then exposed to etoposide. In RNA interference assay, $>90 \%$ of the transfected cells expressed this synthetic E2F1 siRNA, and endogenous expression of E2F1 was effectively suppressed (Fig. 5A and $\mathrm{B}, \mathrm{P}<0.05$ ). However, the inhibition of proliferation (Fig. 5C) and induction of apoptosis (Fig. 5D and E) were almost completely abolished after E2F1 was knocked down in both the TRIM28-silenced group as well as in the si-TRIM28 negative control group which indicated that the promotion of sensitivity to etoposide of TRIM28 siRNA is mediated by E2F1 activation.

\section{Discussion}

Resent studies indicate that TRIM28 is an important regulator of carcinogenesis in several neoplasmas. Yokoe et al (13) demonstrated that TRIM28 provides a survival advantage to gastric cancer cells and is an independent factor for peritoneal dissemination in gastric cancer patients. An increase in TRIM28 expression which was induced by loss of ZBRK1 enhanced the migration and invasion of cervical cancer cells both in vitro and in vivo (10). Addison et al (11) showed that TRIM28 is overexpressed in breast tumors and the depletion of TRIM28 in breast cancer cell lines slowed cell proliferation and inhibited the growth and metastasis of tumor xenografts. Multiple genes linked to tumor progression and metastasis, including PTGS2/COX2, EREG, CD44, MMP1, MMP2, were downregulated in breast cancer cells when TRIM28 was silenced. Our previous study found that TRIM28 was upregulated in NSCLC cell lines and tissues, promoting the growth and survival of lung cancer cells. In this study, we further 
A

B

H\&E ( $\times 200)$ H\& $(\times 200)$

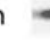

Etoposide

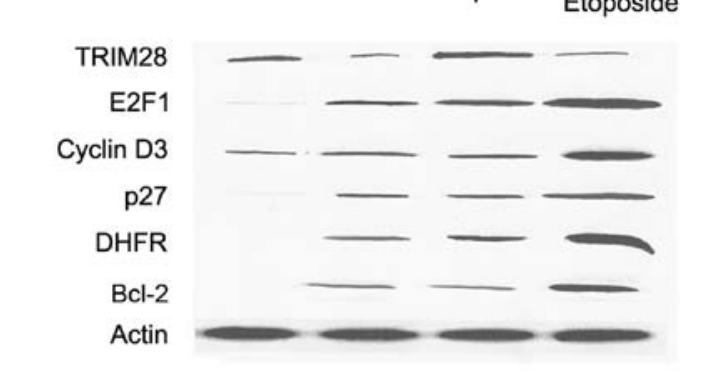

si-control si-TRIM28 Etoposide si-TRIM28+

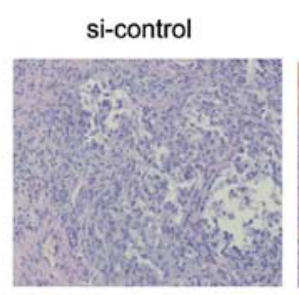

TRIM28 (×200)
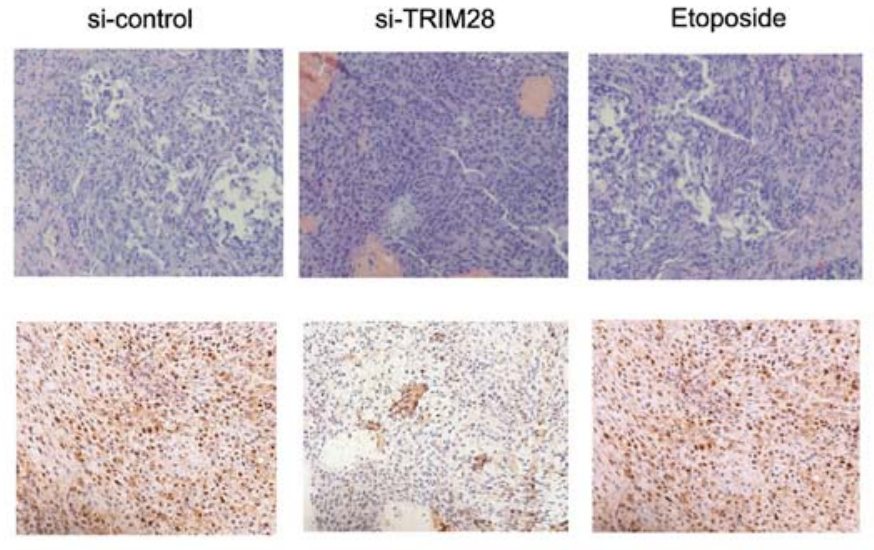

si-TRIM28+Etoposide
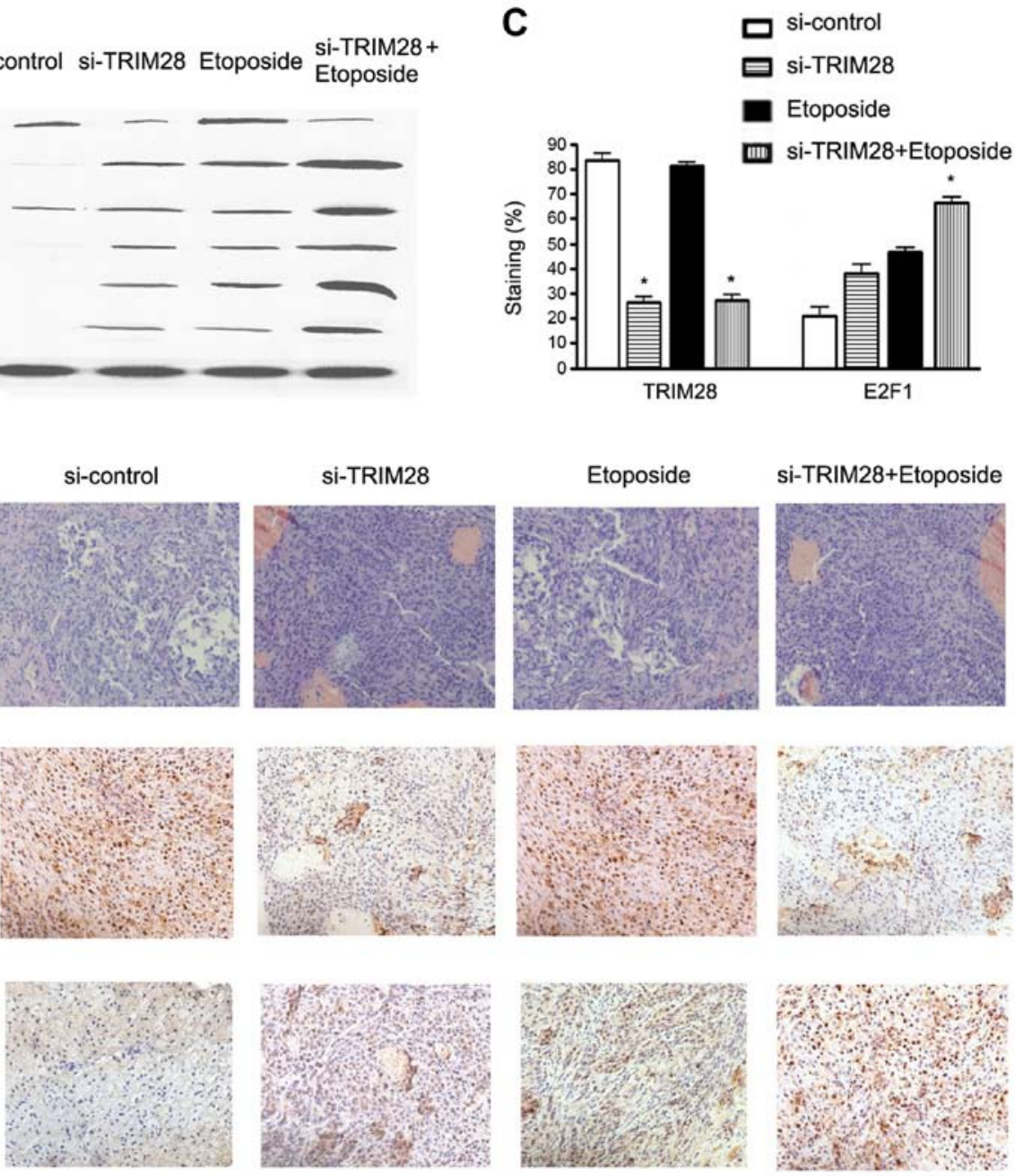

Figure 4. TRIM28 siRNA in combination with etoposide increases the expression of E2F1. (A) Reduction in the expression of E2F1 and target genes in PAa cells treated with TRIM28 siRNA, etoposide or combination of TRIM28 siRNA and etoposide, compared to that in the cells treated with negative control siRNA were determined by western blot analysis. (B and C) Expression of E2F1 was examined by IHC in tumors from the four mouse groups. The tumor cells in the cotreatment group had significantly higher levels of E2F1 expression as compared with those in the control group. $\mathrm{P}<0.05$ in comparison to the si-control group.

confirmed that stable TRIM28 silencing significantly inhibited the growth of PAa cells in DMEM and colony formation ability in soft agar. The proliferation of PAa cells transfected with TRIM28 siRNA decreased by $33.78 \%$ when compared to that noted in the PAa/control-siRNA cells after 4 days by using an MTT assay. Our study also confirmed the antitumor effect of TRIM28 siRNA in nude mice; the growth inhibition was statistically significant compared with that of the controls.

Although a growing number of studies suggest that TRIM28 plays a role in oncogenesis, the underlying mechanism of TRIM28 remains unclear. Following DNA damage, TRIM28 is rapidly phosphorylated and localized to sites of DNA strand breaks in the nucleus (7). In addition, HP1-dependent regulation of TRIM28 influenced DNA repair in heterochromatin (18). TRIM28 stimulated formation of the p53-HDAC1 complex and inhibited p53 acetylation by interacting with MDM2. Depletion of endogenous TRIM28 expression by RNAi stimulated p53 transcriptional activity, sensitized p53 response to DNA damage, and increased apoptosis (19). Kamitani et al (20) found that TRIM28 associated with endogenous STAT1 repressed the interferon (IFN)-mediated signaling pathway. A
siRNA-mediated reduction in TRIM28 expression enhanced IFN-induced STAT1-dependent IRF-1 gene expression. A recent study suggested that FIK acts as a bridging molecule to link FOXP3 with TRIM28. Disruption of the FOXP3-FIKTRIM28 complex in Tregs restored expression of FOXP3-target genes and abrogated the suppressor activity of Tregs (21). These signaling pathways where TRIM28 plays a role were associated with apoptosis in tumor genesis. The present study showed that TRIM28 silencing increased apoptosis in vitro and in vivo. We observed that cell apoptosis occurred after the NSCLC cell line PAa was transfected with TRIM28 siRNA (apoptotic cells, $3.03 \pm 0.25 \%$ ), significantly higher than the control group. The degree of apoptosis in the tumors treated with TRIM28 siRNA $(24.65 \pm 4.05)$ was much higher than that in the control siRNA group (8.20 $\pm 2.11, \mathrm{P}=0.004)$. Moreover, we observed that the expression of E2F1 and several target genes was increased in the PAa cells transfected with TRIM28 siRNA, and E2F1 expression was upregulated in tumors from the TRIM28 siRNA treatment group mice. These results suggest that TRIM28 regulates apoptosis induced by the E2F1 signaling pathway, in which TRIM28 binds the E2F1 transcription factor in a 
A

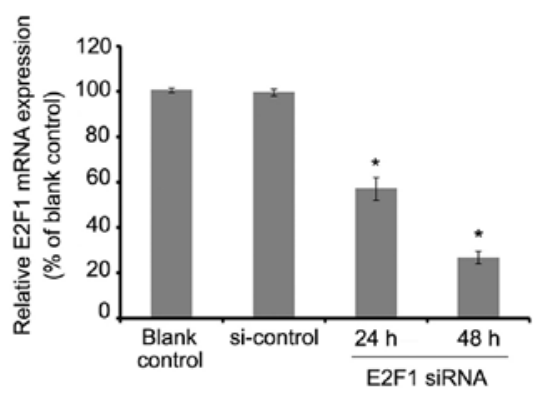

C

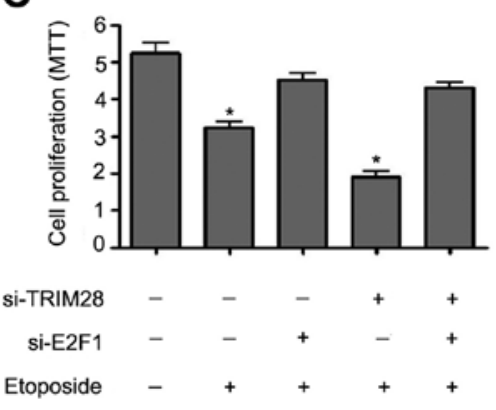

B
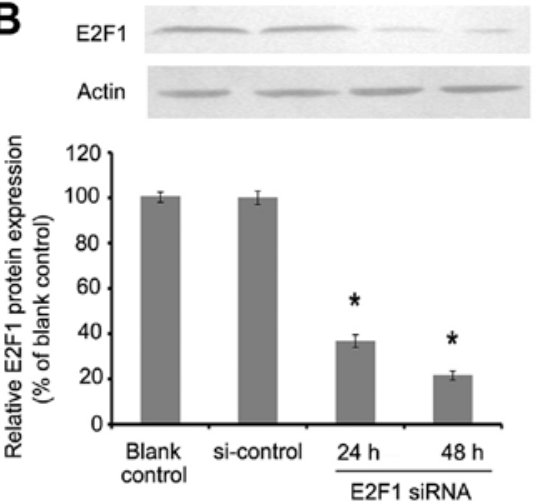

E

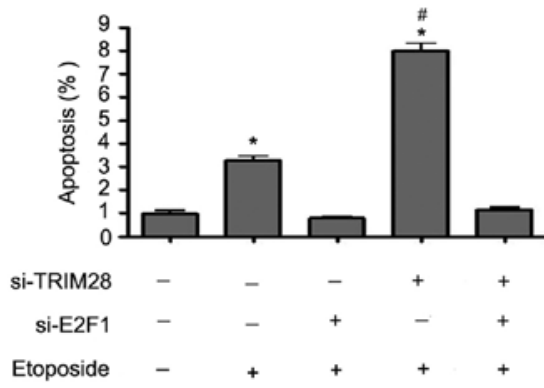

D

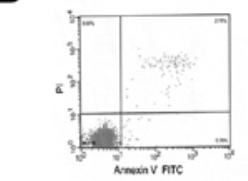

si-TRIM28

si-E2F1

Etoposide
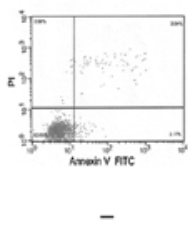

$-$

$+$
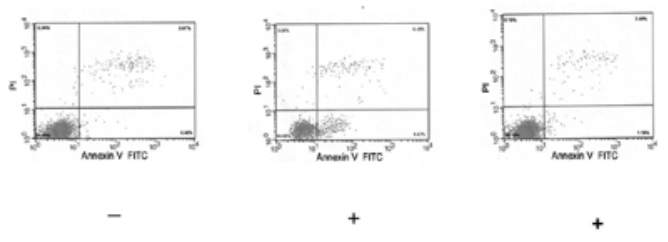

$+$

$+$

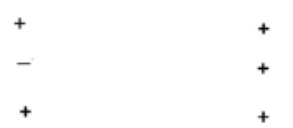

Figure 5. Affects on cell proliferation and apoptosis by TRIM28 siRNA in combination with etoposide were almost completely abolished following E2F1 knockdown. (A and B) Endogenous expression of E2F1 was effectively suppressed by E2F1 RNA interference and confirmed by real-time PCR (A) and western blot analysis (B). $\mathrm{P}<0.05$ in comparison to the negative control group. (C) Cell proliferation was analyzed by MTT assay. The cell proliferation was almost completely abolished by si-E2F1 both in the TRIM28-silenced group as well as in the si-TRIM28 negative control group. ${ }^{*} \mathrm{P}<0.05$ in comparison to negative control group. (D and E) Apoptosis was examined by flow cytometric analysis. The apoptosis was also abolished by si-E2F1 both in the si-TRIM28 group as well as in the si-TRIM28 negative group. " $\mathrm{P}<0.05$ in comparison to the negative control group. ${ }^{\#} \mathrm{P}<0.05$ in comparison to the etoposide treatment alone group.

PRb-independent fashion and inhibits E2F1 activity (16). In our study, the E2F1 acetylation level increased, E2F1 transcription activity was promoted, and apoptosis response was sensitized after the endogenous TRIM28 was stably interfered.

Etoposide is a first-line cytotoxic drug for advanced NSCLC, and belongs to the topoisomerase inhibitor drug class. Etoposide formes a ternary complex with DNA and the topoisomerase II enzyme (which aids in DNA unwinding), prevents re-ligation of the DNA strands, and by doing so causes DNA strands to break. Cancer cells rely on this enzyme more than healthy cells, since they divide more rapidly. Therefore, this causes errors in DNA synthesis and promotes apoptosis of the cancer cells. Numerous studies have shown that E2F1 is critical for etoposide-induced apoptosis. E2F1 protein levels were increased following treatment with the DNA damaging agent etoposide. In contrast, overexpression of E2F1 protein in tumor cell lines led to enhanced cytotoxicity of etoposide, resulting from enhanced apoptosis (17). Nip et al (22) reported that E2F1 overexpression in myeloid cells preferentially sensitized cells to apoptosis when they were treated with etoposide, which was independent of p53 accumlation. Dong et al (23) used two human melanoma cell lines treated with etoposide alone or in combination with adenoviral vectors expressing E2F1 to evaluate the effect of E2F1 expression on etoposide sensitivity. Cotreatment with Ad-E2F1 and low concentrations of etoposide markedly sensitized melamoma cells to apoptotic cell death. Topoisomerase II inhibitors also cooperated with Ad-E2F1 to enhance antitumor activity in an in vivo model using xenografts in nude mice. In the present study, we observed that etoposide inhibited the growth and promoted the apoptosis of NSCLC cells in vitro and in vivo, and upregulated the expression of E2F1 and its target genes.

These results indicated that overexpression of $\mathrm{E} 2 \mathrm{~F} 1$ protein promoted apoptosis and enhanced the cytotoxicity of etoposide. In addition, reduction of TRIM28 upregulated E2F1 transcription activity; thus we speculated that TRIM28 knockdown 
would increase the sensitivity to etoposide in NSCLC cells. To determine the hypothesis, we examined the antitumor effects of TRIM28 siRNA in combination with etoposide in vitro and in vivo. Cotreatment with TRIM28 siRNA and etoposide inhibited the growth and proliferation more effectively in PAa cells and nude mice. We further showed that the largest number of apoptotic cells was observed in the PAa cells and mouse tumors treated with the combination of TRIM28 siRNA and etoposide. However, the inhibition of proliferation and induction of apoptosis were almost completely abolished after E2F1 was knocked down in the cells cotreated with TRIM28 siRNA and etoposide, which indicated that the promotion of sensitivity to etoposide of TRIM28 siRNA is mediated by E2F1 activation. A similar study was conducted by Okamoto et al (24). The authors examined the role of TRIM28 in p53 activation after the treatment of cells with different types of chemotherapeutic agents. TRIM28 reduction markedly enhanced the induction of p21, a product of the p53 target gene, after treatment with actinomycin D or $\gamma$-irradiation. Treatment with actinomycin D augmented the interaction of p53 with Mdm2 and TRIM28. Furthermore, TRIM28 reduction in the actinomycin D-treated cells facilitated cell cycle arrest and negatively affected clonal cell growth. Thus, the reduction in TRIM28 levels promoted p53-dependent p21 induction and inhibited cell proliferation in actinomycin D-treated cells. These results suggest that TRIM28 recruits many proteins involved in gene silencing and functions as an integral part of a co-repressor complex. TRIM28 may serve as a therapeutic target against cancer in combination with actinomycin D or etoposide.

\section{Acknowledgements}

This study was supported by the Hebei Natural Science Foundation (H2015406014); the Hebei Talent Engineering Training Funded Research Projects (A2016002085); the Heibei University Science and Technology Research Project (QN20131010); and the Hebei provincial focused discipline.

\section{References}

1. Jemal A, Bray F, Center MM, Ferlay J, Ward E and Forman D: Global cancer statistics. CA Cancer J Clin 61: 69-90, 2011.

2. Maghfoor I and Perry MC: Lung cancer. Ann Saudi Med 25: $1-12,2005$.

3. Hanna N, Neubauer M, Yiannoutsos C, McGarry R, Arseneau J, Ansari R, Reynolds C, Govindan R, Melnyk A, Fisher W, et al; Hoosier Oncology Group; US Oncology: Phase III study of cisplatin, etoposide, and concurrent chest radiation with or without consolidation docetaxel in patients with inoperable stage III non-small-cell lung cancer: The Hoosier Oncology Group and US Oncology. J Clin Oncol 26: 5755-5760, 2008.

4. Messerschmidt DM, de Vries W, Ito M, Solter D, FergusonSmith A and Knowles BB: Trim28 is required for epigenetic stability during mouse oocyte to embryo transition. Science 335 : 1499-1502, 2012.

5. Barde I, Rauwel B, Marin-Florez RM, Corsinotti A, Laurenti E, Verp S, Offner S, Marquis J, Kapopoulou A, Vanicek J, et al: A KRAB/KAP1-miRNA cascade regulates erythropoiesis through stage-specific control of mitophagy. Science 340: 350-353, 2013.
6. Pfeifer GP: Protein phosphatase PP4: Role in dephosphorylation of KAP1 and DNA strand break repair. Cell Cycle 11: 2590-2591, 2012.

7. White DE, Negorev D, Peng H, Ivanov AV, Maul GG and Rauscher FJ III: KAP1, a novel substrate for PIKK family members, colocalizes with numerous damage response factors at DNA lesions. Cancer Res 66: 11594-11599, 2006.

8. Gilmore-Hebert M, Ramabhadran R and Stern DF: Interactions of ErbB4 and Kap1 connect the growth factor and DNA damage response pathways. Mol Cancer Res 8: 1388-1398, 2010.

9. Hu C, Zhang S, Gao X, Gao X, Xu X, Lv Y, Zhang Y, Zhu Z, Zhang C, Li Q, et al: Roles of Kruppel-associated box (KRAB)-associated co-repressor KAP1 Ser-473 phosphorylation in DNA damage response. J Biol Chem 287: 18937-18952, 2012.

10. Lin LF, Li CF, Wang WJ, Yang WM, Wang DD, Chang WC, Lee WH and Wang JM: Loss of ZBRK1 contributes to the increase of KAP1 and promotes KAP1-mediated metastasis and invasion in cervical cancer. PLoS One 8: e73033, 2013.

11. Addison JB, Koontz C, Fugett JH, Creighton CJ, Chen D, Farrugia MK, Padon RR, Voronkova MA, McLaughlin SL, Livengood RH, et al: KAP1 promotes proliferation and metastatic progression of breast cancer cells. Cancer Res 75: 344-355, 2015.

12. Fitzgerald S, Sheehan KM, O 'Grady A, Kenny D, O 'Kennedy R, Kay EW and Kijanka GS: Relationship between epithelial and stromal TRIM28 expression predicts survival in colorectal cancer patients. J Gastroenterol Hepatol 28: 967-974, 2013.

13. Yokoe T, Toiyama Y, Okugawa Y, Tanaka K, Ohi M, Inoue Y, Mohri Y, Miki C and Kusunoki M: KAP1 is associated with peritoneal carcinomatosis in gastric cancer. Ann Surg Oncol 17: 821-828, 2010.

14. Zhang L, Zhu C, Guo Y, Wei F, Lu J, Qin J, Banerjee S, Wang J, Shang H, Verma SC, et al: Inhibition of KAP1 enhances hypoxia-induced Kaposi 's sarcoma-associated herpesvirus reactivation through RBP-Jк. J Virol 88: 6873-6884, 2014.

15. Liu L, Zhao E, Li C, Huang L, Xiao L, Cheng L, Huang X, Song Y and Xu D: TRIM28, a new molecular marker predicting metastasis and survival in early-stage non-small cell lung cancer. Cancer Epidemiol 37: 71-78, 2013.

16. Wang C, Rauscher FJ III, Cress WD and Chen J: Regulation of E2F1 function by the nuclear corepressor KAP1. J Biol Chem 282: 29902-29909, 2007.

17. Meng RD, Phillips P and El-Deiry WS: p53-independent increase in E2F-1 expression enhances the cytotoxic effects of etoposide and of adriamycin. Int J Oncol 14: 5-14, 1999.

18. White D, Rafalska-Metcalf IU, Ivanov AV, Corsinotti A, Peng H, Lee SC, Trono D, Janicki SM and Rauscher FJ III: The ATM substrate KAP1 controls DNA repair in heterochromatin: Regulation by HP1 proteins and serine $473 / 824$ phosphorylation. Mol Cancer Res 10: 401-414, 2012.

19. Wang C, Ivanov A, Chen L, Fredericks WJ, Seto E, Rauscher FJ III and Chen J: MDM2 interaction with nuclear corepressor KAP1 contributes to p53 inactivation. EMBO J 24: 3279-3290, 2005.

20. Kamitani S, Ohbayashi N, Ikeda O, Togi S, Muromoto R, Sekine Y, Ohta K, Ishiyama $\mathrm{H}$ and Matsuda T: KAP1 regulates type I interferon/STAT1-mediated IRF-1 gene expression. Biochem Biophys Res Commun 370: 366-370, 2008.

21. Huang C, Martin S, Pfleger C, Du J, Buckner JH, Bluestone JA, Riley JL and Ziegler SF: Cutting Edge: A novel, human-specific interacting protein couples FOXP3 to a chromatin-remodeling complex that contains KAP1/TRIM28. J Immunol 190: 4470-4473, 2013.

22. Nip J, Strom DK, Fee BE, Zambetti G, Cleveland JL and Hiebert SW: E2F-1 cooperates with topoisomerase II inhibition and DNA damage to selectively augment p53-independent apoptosis. Mol Cell Biol 17: 1049-1056, 1997.

23. Dong YB, Yang HL, Elliott MJ and McMasters KM: Adenovirus-mediated E2F-1 gene transfer sensitizes melanoma cells to apoptosis induced by topoisomerase II inhibitors. Cancer Res 62: 1776-1783, 2002.

24. Okamoto K, Kitabayashi I and Taya Y: KAP1 dictates p53 response induced by chemotherapeutic agents via Mdm2 interaction. Biochem Biophys Res Commun 351: 216-222, 2006. 